\title{
Paraneoplastic polyarthritis in association with metastatic neuroendocrince tumour of the adrenal gland
}

\section{Sahana Shetty ${ }^{1}$, Julie Hephzibah², Bidyut Borah ${ }^{1}$, Deepak Burad ${ }^{3}$, Vishnu Chandrakumar ${ }^{3}$,} Mazhuvanchary Jacob Paul ${ }^{4}$, Nihal Thomas ${ }^{1}$

1. Department of Endocrinology, Diabetes \& Metabolism, Christian Medical College, Vellore, India

2. Department of Nuclear Medicine, Christian Medical College, Vellore, India

3. Department of General Pathology, Christian Medical College, Vellore, India

4. Department of Endocrine Surgery, Christian Medical College, Vellore, India

\section{CASE STUDY}

Please cite this paper as: Shetty S, Hephzibah J, Borah B, Burad D, Chandrakumar V, Paul MJ, Thomas N. Paraneoplastic polyarthritis in association with metastatic neuroendocrine tumour of the adrenal gland. AMJ 2014, 7, 8, 345-349. http://doi.org/10.21767/AMJ.2014.2113

Corresponding Author:

Dr Nihal Thomas

Professor and Head, Department of Endocrinology, Diabetes and Metabolism \& Vice-Principal (Research), Christian Medical College, Vellore-632004, India. Email: nihal_thomas@yahoo.com

\section{ABSTRACT}

Paraneoplastic polyarthritis is a rare manifestation described in association with various solid tumours. We describe the clinical presentation, diagnostic evaluation, differential diagnosis, and management of a 28-year-old woman who presented with fever, weight loss, and symmetrical polyarthritis, subsequently diagnosed to have a metastatic neuroendocrine tumour of the adrenal gland with paraneoplastic polyarthritis. Paraneoplastic polyarthritis must be considered in polyarthritis unexplained by common aetiologies. The unusual presentation of this case, alerts us about the atypical presentation of these tumours. To the best of our knowledge, this is the first case study of a neuroendocrine tumour presenting as paraneoplastic polyarthritis.

\section{Key Words}

Paraneoplastic polyarthritis, neuroendocrine tumour, metastasis

\section{Implications for Practice:}

\section{What is known about this subject?}

Paraneoplastic polyarthritis is an uncommon cause of polyarthritis, described in association with various solid tumours.

\section{What new information is offered in this case study?} In this case report, we describe paraneoplastic polyarthritis in association with a metastatic neuroendocrine tumour of the adrenal gland, which is an unusual presentation.

\section{What are the implications for research, policy, or practice?}

Paraneoplastic polyarthritis must be considered if the polyarthritis cannot be explained by common aetiologies. The unusual presentation of this case also alerts us about the atypical presentations of neuroendocrine tumours.

\section{Background}

Paraneoplastic syndromes are manifestations occurring either during the course of malignancy or preceding the clinical evidence of neoplasm and are not the result of direct tumour invasion or compression. ${ }^{1}$

Polyarthritis is a common clinical symptom, which can be secondary to a number of aetiologies, including inflammatory polyarthritis-like rheumatoid arthritis, spondyloarthritis, systemic infections, vasculitic syndromes, connective tissue disorders, crystal-induced arthropathy, and paraneoplastic syndrome. ${ }^{2}$

Paraneoplastic polyarthritis is a rare paraneoplastic presentation described in association with various tumours. Paraneoplastic polyarthritis is a rare entity with published literature restricted largely to case series and case reports. In this case study, we describe paraneoplastic polyarthritis in association with a metastatic neuroendocrine tumour of the adrenal gland, which is an unusual presentation. 
Neuroendocrine tumours (NETs) are neoplasms composed of cells that harbour the unique ability to synthesise, store, and secrete a variety of metabolically active substances, peptides and amines, which can cause distinct clinical syndromes.

Neuroendocrine tumours are associated with various paraneoplastic manifestations due to secretion of various mediators like adrenocorticotrophic hormone (ACTH), corticotrophin-releasing hormone (CRH), parathyroid hormone (PTH), parathyroid hormone related protein (PTHrP), growth hormone (GH), growth hormone releasing hormone $(\mathrm{GHRH})$, calcitonin, calcitonin gene related peptide (CGRP), cytokines etc. Awareness of these associations is important for proper diagnosis and management as they improve with the treatment of the underlying neoplasm. ${ }^{3}$

\section{Case details}

A 28-year-old woman presented with fever and peripheral symmetric polyarthritis involving the small and large joints, of seven months duration. The polyarthritis was insidious in onset with painful swelling of bilateral knee joint, followed by shoulder, elbow, wrist, ankle, and the small joints of the hand with gradual development of flexion contractures at the knees and elbows. She had an uneventful vaginal delivery eight months previously. She also reported a marked weight loss of $25 \mathrm{~kg}$ following delivery. There was no history of skin rashes, photosensitivity, red eye, oral ulcers, sore throat, diarrhoea, urethritis, or tick bite. On examination, she was pale and emaciated with a body mass index of $15 \mathrm{~kg} / \mathrm{m}^{2}$; she was febrile with a temperature of $38.8^{\circ} \mathrm{C}$. There was dacytilitis involving both hands (Figure 1 ).

\section{Figure 1: Dactylitis}

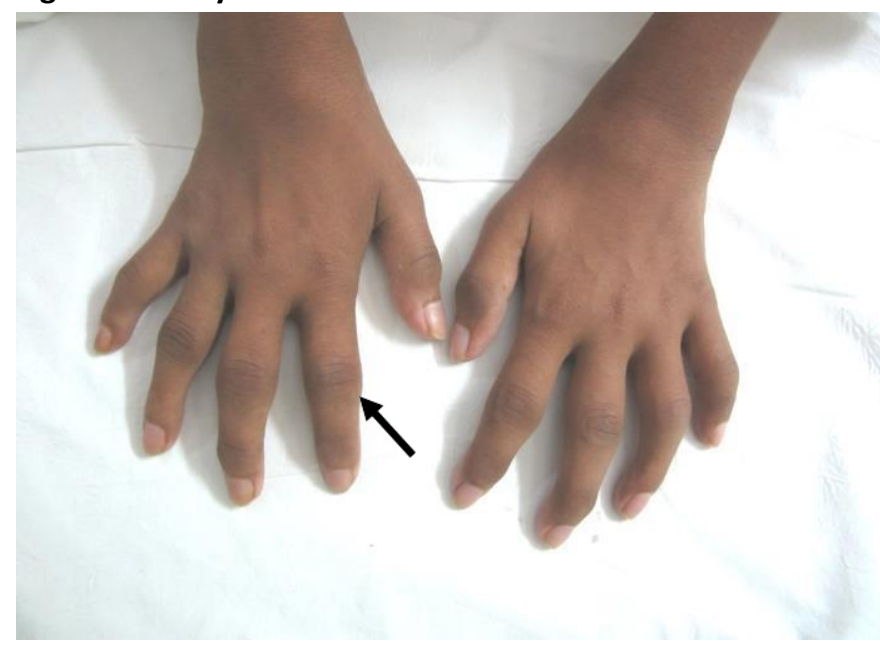

There was swelling, warmth, and tenderness in bilateral wrists, elbows, knees, ankles, and the small joints of the hands and feet with painful restriction of movements. There were flexion contractures at the inter-phalangeal joints, elbows, and knees. There was significant generalised lymphadenopathy and moderate hepatosplenomegaly. The differential diagnoses considered were rheumatoid arthritis, infectious polyarthritis, vasculitic syndromes, subacute bacterial endocarditis, and paraneoplastic polyarthritis.

Her haemogram revealed a normocytic anaemia with a haemoglobin count of $9.8 \mathrm{gm} / \mathrm{dl}$ (Normal 13-15), rest of cell lines were within normal limits [WBC count of $9,200 / \mathrm{mm}^{3}$ (Normal 4,000-11,000), platelet count of $310,000 / \mathrm{mm}^{3}$ (Normal 150,000-400,000)]. Her erythrocyte sedimentation rate (ESR) was $88 \mathrm{~mm}$ at the end of one hour (Normal up to $20 \mathrm{~mm})$.

Her biochemical parameters were as follows: serum creatinine: $51 \mu \mathrm{mol} / \mathrm{L}$ (Normal 51-97); serum sodium: $137 \mathrm{mmol} / \mathrm{L}$ (Normal 135-145); serum potassium: $4.1 \mathrm{mmol} / \mathrm{L}$ (Normal 3.5-5); total bilirubin: $9 \mathrm{mmol} / \mathrm{L}$ (Normal 8.5-17); serum albumin: 37g/L (Normal 4.5-7); AST: $18 \mathrm{U} / \mathrm{L}$ (Normal 8-40); ALT: 5U/L (Normal 5-35); alkaline phosphatase: $175 \mathrm{U} / \mathrm{L}$ (Normal 40-125); serum uric acid: $440 \mu \mathrm{mol} / \mathrm{L}$ (Normal 140-360); serum LDH: 385U/L (Normal 225-460). Her immunological workup showed an elevated $C$ reactive protein (CRP) of $46.7 \mathrm{mg} / \mathrm{L}$ (Normal <6). However, her rheumatoid factor (RA), ANA, ds DNA, AntiCCP, C-ANCA, P-ANCA, Combined ENA (extractable nuclear antigen) were negative. Her serum complement $\mathrm{C} 3$ and $\mathrm{C} 4$ levels were within normal limits. Her screening for hepatitis serology was negative. Her chest $\mathrm{x}$-ray was normal.

The CT abdomen was done as a part of a work-up for pyrexia of unknown origin (PUO) with undifferentiated polyarthritis and weight loss as most of the other aetiological work-up (infectious, rheumatological, vasculitic, and crystal arthropathies) was negative. CT of the abdomen showed multiple large vascular lesions with nonenhancing necrotic centre in the liver (Figure 2) with larger lesions measuring about $11 \times 8.4 \mathrm{~cm}$. In addition, a vascular lesion of $1.8 \times 2.3 \mathrm{~cm}$ with a necrotic centre in the left adrenal gland was also seen. The differential diagnoses considered at this stage were granulomatous diseases like histoplasmosis and malignant neoplasms arising either from the adrenal cortex or medulla with metastasis. Further work-up was done to assess the functional status of adrenal mass which was within normal limits: 8am cortisol of 413nmol/L (Normal 171-618); 24-hour urinary cortisol: 200nmol/L (Normal <250); post-1mg dexamethasone: $33 \mathrm{nmol} / \mathrm{L}$ (Normal <50); DHEAS: 3.1 $\mu \mathrm{mol} / \mathrm{L}$ (Normal 2.1-10); 24-hour urinary metanephrines: 700nmol/L (Normal <350); and normetanephrines: 1,640 nmol/L (Normal <600). 
To obtain a tissue diagnosis, a biopsy of the hepatic lesion was done under ultrasound guidance. Histopathology examination showed a nest of tumour cells (Figure 3 ).

Figure 2: CT abdomen: (a) axial section arterial phase; (b) coronal section venous phase

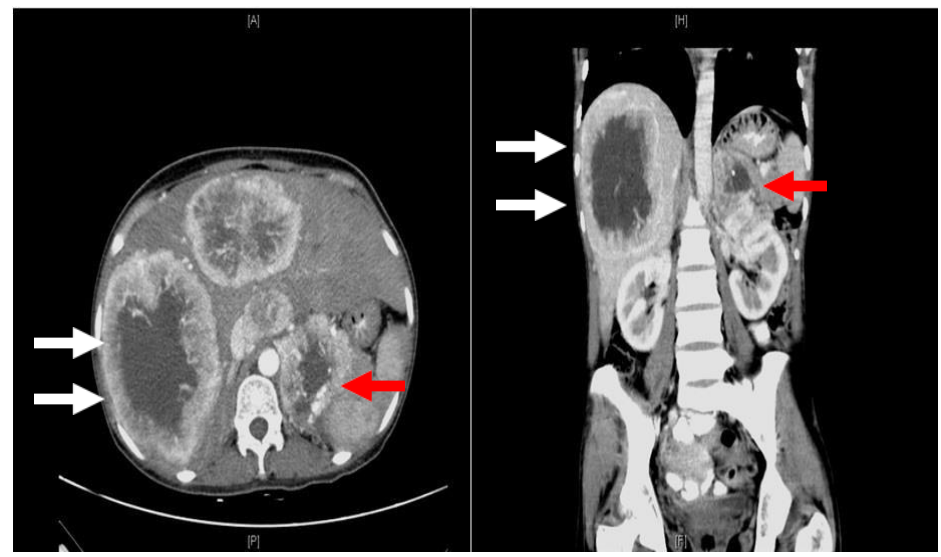

White arrows denote large vascular lesions with nonenhancing necrotic centre in the liver with larger lesions measuring about $11 \times 8.4 \mathrm{~cm}$. Red arrows showing a $1.8 \times$ $2.3 \mathrm{~cm}$ vascular lesion with a necrotic centre in the left adrenal gland

\section{Figure 3: Histopathology}
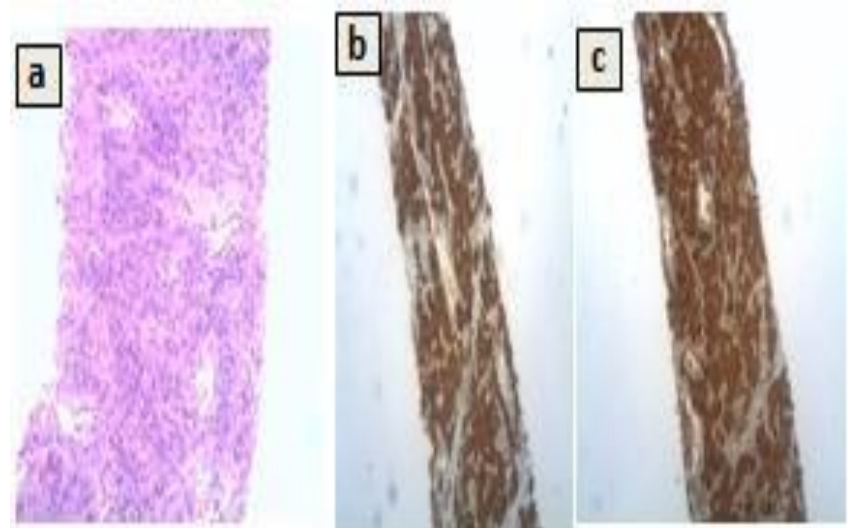

(a) Haematoxylin and Eosin $\times 200$ showing a nested arrangement of tumour cells (zellballen appearance). Immunohistochemistry $x 100$ positive for (b) synaptophysin and (c) chromogranin.

Immunohistochemical staining for synaptophysin and chromogranin were positive (and cytokeratin negative), suggestive of neuroendocrine differentiation with a MIB1 index of 15-20 per cent.

An I $^{131}$-MIBG (Meta-iodo-benzyl-guanidine) scintigraphy displayed an abnormal tracer uptake in the left suprarenal region, and a large area of circumferential tracer activity with central photopenia was seen in the liver (Figure 4). Thus, a diagnosis of a metastatic neuroendocrine tumour of left adrenal with liver and lung metastasis was established, and the polyarthritis was considered as a paraneoplastic presentation.

\section{Figure 4: I $^{131}$-MIBG whole-body scan}

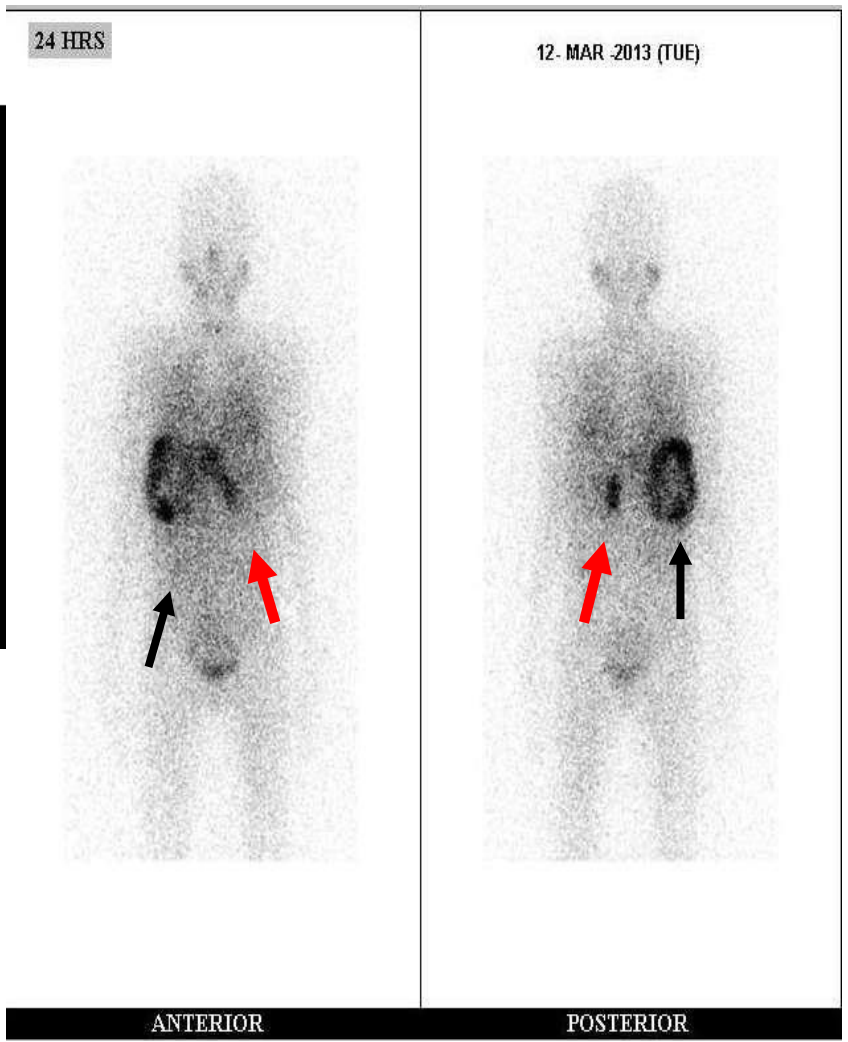

Abnormal tracer uptake in the left suprarenal region (red arrow) and a large area of circumferential tracer activity with central photopenia in the liver (black arrow)

The patient was not considered for surgical intervention, in view of multiple large metastatic hepatic and lung lesions and her relatively suboptimal general condition. She was treated with $I^{131}$ MIBG Ablation $(100 \mathrm{mCi})$ and was also given Octreotide-LAR 20mg every three weeks. Glucocorticoids and physiotherapy were offered for symptomatic relief of polyarthritis. At three months of follow-up, she had complete resolution of her arthritis. However, hepatic and adrenal lesions remained static on reimaging.

\section{Discussion}

Paraneoplastic polyarthritis is a rare paraneoplastic manifestation associated with some solid tumours like ovarian, lung, gastric, colon, breast, and laryngeal neoplasms. Paraneoplastic polyarthritis is a diagnosis of exclusion. Most of the aetiologies of polyarthritis can be diagnosed based on the mode of presentation, patterns of articular involvement, associated systemic symptoms, radiological presentation, inflammatory markers, and autoantibodies. 
The age of presentation differs depending on the underlying primary malignancy and there appears to be no genderrelated predilection. The age of presentation in various reported cases ranged from 43-76 years with a mean age of 61 years. In most published literature, paraneoplastic polyarthritis preceded the diagnosis of cancer between 120 months and most malignancies were detected within three months of polyarthritis. ${ }^{2}$

Symptoms usually improve with the treatment of the underlying neoplasm and recurrence indicates relapse of malignancy. In our case, polyarthritis improved with steroids and treatment of the underlying neuroendocrine tumour with somatostatin analogues and MIBG therapy. In a review by Zupancic et al., 12 out of 13 cases demonstrated an improvement in polyarthritis after treatment of the underlying malignancy either following surgery or chemotherapy. ${ }^{1}$

The pathogenesis of paraneoplastic polyarthritis has not been fully understood. Circulating immune complexes and platelet activating factors have been postulated to cause sterile inflammatory response in the synovium leading to polyarthritis. ${ }^{1}$

Other mechanisms that have been proposed involve a molecular mimicry between tumour antigens and the synovium, an immunological response against the tumour and an autoimmune phenomenon secondary to the reactive lymphocytes from the lymph nodes that drain the tumour. ${ }^{5}$

Paraneoplastic polyarthritis may improve with the treatment of the underlying neoplasm, which might be difficult in metastatic malignancies. Specific to our patient's management, the malignant nature of the phaeochromocytoma is defined by the presence of metastasis at non-chromaffin sites distinct from the primary tumour. ${ }^{6}$

Treatment options for malignant phaeochromocytoma include surgical debulking of both primary and metastatic

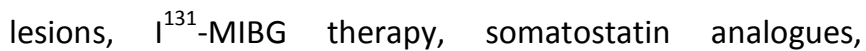
chemotherapy, radiotherapy, and tumour-directed therapies like radiofrequency ablation, arterial embolization, and cryoablation mainly directed at metastatic lesions. Surgery is considered the mainstay of palliative therapy if feasible. In inoperable cases, $1^{131}$-MIBG therapy has been described to be most effective in patients with positivity on MIBG scintigraphy. ${ }^{7}$

\section{Conclusion}

This case study emphasises the importance of clinical suspicion of paraneoplastic polyarthritis in new onset polyarthritis, which is unexplained by the common aetiologies. The unusual presentation witnessed here alerts us about the atypical presentations of these tumours. To the best of our knowledge, this is the first case report of a neuroendocrine tumour presenting as paraneoplastic polyarthritis.

\section{References}

1. Zupancic M, Annamalai A, Brenneman J, Ranatunga S Migratory Polyarthritis as a Paraneoplastic Syndrome. J Gen Intern Med. 2008; 23(12):2136-9.

2. Ruddy S, Harris E, Sledge C. Kelly's Textbook of Rheumatology, 6th ed. Philadelphia: WB Saunders Company. 2001;967-1000.

3. Kaltsas G, Androulakis I, Herder WW, Grossman AB. Paraneoplastic syndromes secondary to neuroendocrine tumours. Endocr Relat Cancer. 2010;17(3):173-93.

4. Bradley JD, Pinals RS. Carcinoma polyarthritis: role of immune complexes in pathogenesis. J Rheumatology. 1983; 1058:26-8.

5. Fam AG. Paraneoplastic rheumatic syndromes. Baill Clin Rheum. 2000;14:3515-33.

6. Chrisoulidou A, Kaltsas G, Ilias I, Grossman AB. The diagnosis and management of malignant phaeochromocytoma and paraganglioma. EndocrineRelated Cancer 2007; 14:569-585.

7. Scholz T, Eisenhofer G, Pacak K, Dralle H, Lehner H. Clinical review: Current Treatment of Malignant Pheochromocytoma. J Clin Endocrinol Metab. 2007; 92(4):1217-25.

\section{PEER REVIEW}

Not commissioned. Externally peer reviewed.

\section{CONFLICTS OF INTEREST}

The authors declare that they have no competing interests.

\section{ETHICS COMMITTEE APPROVAL}

Ethical committee approval was obtained from the Institutional Review Board (IRB) of Christian Medical College, Vellore, India, with the IRB reference number no 9006.

\section{PATIENT CONSENT}

The authors, Shetty S, Hephzibah J, Borah B, Burad D, Chandrakumar V, Paul MJ, Thomas N, declare that: 
1. They have obtained written, informed consent for the publication of the details relating to the patient(s) in this report.

2. All possible steps have been taken to safeguard the identity of the patient(s).

3. This submission is compliant with the requirements of local research ethics committees. 